\title{
Entanglement spectrum of the Heisenberg XXZ chain near the ferromagnetic point
}

\author{
Vincenzo Alba ${ }^{1}$ \\ ${ }^{1}$ Max-Planck-Institut für Physik komplexer Systeme, Nöthnitzer Straße 38, D-01187 \\ Dresden, Germany \\ Masudul Haque ${ }^{2}$ \\ 2 Max-Planck-Institut für Physik komplexer Systeme, Nöthnitzer Straße 38, D-01187 \\ Dresden, Germany
}

\author{
Andreas M. Läuchli ${ }^{3}$ \\ ${ }^{3}$ Institute für Theoretische Physik, Universität Innsbruck, A-6020 Innsbruck, Austria
}

\begin{abstract}
.
We study the entanglement spectrum (ES) of a finite XXZ spin- $\frac{1}{2}$ chain in the limit $\Delta \rightarrow-1^{+}$for both open and periodic boundary conditions. At $\Delta=-1$ (ferromagnetic point) the model is equivalent to the Heisenberg ferromagnet and its degenerate ground state manifold is the $S U(2)$ multiplet with maximal total spin. Any state in this so-called "symmetric sector" is an equal weight superposition of all possible spin configurations. In the gapless phase at $\Delta>-1$ this property is progressively lost as one moves away from the $\Delta=-1$ point. We investigate how the ES obtained from the states in this manifold reflects this change, using exact diagonalization and Bethe ansatz calculations. We find that in the limit $\Delta \rightarrow-1^{+}$most of the ES levels show divergent behavior. Moreover, while at $\Delta=-1$ the ES contains no information about the boundaries, for $\Delta>-1$ it depends dramatically on the choice of boundary conditions. For both open and periodic boundary conditions the ES exhibits an elegant multiplicity structure for which we conjecture a combinatorial formula. We also study the entanglement eigenfunctions, i.e. the eigenfunctions of the reduced density matrix. We find that the eigenfunctions corresponding to the non diverging levels mimic the behavior of the state wavefunction, whereas the others show intriguing polynomial structures. Finally we analyze the distribution of the ES levels as the system is detuned away from $\Delta=-1$.
\end{abstract}




\section{Introduction}

Entanglement is one of the most fascinating concepts in quantum mechanics and the application of entanglement related ideas to condensed matter in recent years has boosted a huge interdisciplinary effort [1]. In particular there has been a growing interest in studying the properties of the so called entanglement spectrum (ES) $[2,3,4,5,6,7,8,9,10]$. The original motivation for the study of the ES was that the ES lies at the heart of the density matrix renormalization group (DMRG) algorithm [2]. More recently, the ES has attracted a lot of attention because of its relation to low-energy boundary modes in topological phases [3].

Considering a bipartition of a system into two parts $A$ and $B$, the entanglement spectrum (ES), $\left\{\xi_{i}\right\}$, is defined in terms of the Schmidt decomposition

$$
|\psi\rangle=\sum_{i} e^{-\xi_{i} / 2}\left|\psi_{i}^{A}\right\rangle \otimes\left|\psi_{i}^{B}\right\rangle
$$

where $|\psi\rangle$ is the state of the system, and $\left|\psi_{i}^{A}\right\rangle\left(\left|\psi_{i}^{B}\right\rangle\right)$ form an orthonormal basis for the subsystem $A(B)$. The $\mathrm{ES}\left\{\xi_{i}=-\log \lambda_{i}\right\}$ can also be thought of in terms of the eigenvalues $\left\{\lambda_{i}\right\}$ of the reduced density matrix $\rho_{A}$ obtained after tracing out the $B$ part of the system density matrix $|\psi\rangle\langle\psi|$.

In this work we study the ES of the finite spin- $\frac{1}{2}$ anisotropic Heisenberg (XXZ) chain in the vicinity of the ferromagnetic point $\Delta=-1$. (Here $\Delta$ is the anisotropy parameter.) In particular we focus on the transformation from the gapless phase at $-1<\Delta \leq 1$, described by a $c=1$ conformal field theory (CFT), to the $\Delta=-1$ point. In the gapless phase (except for the free fermion point $\Delta=0$ where many results are available [2]) only the distribution of the ES levels is known from conformal invariance [10]. On the other hand at $\Delta=-1$ the system is not conformal invariant and a dramatic change occurs. This is reflected in a peculiar behavior of usual entanglement related quantities such as the Renyi entropies and the concurrence $[11,12,13]$. In particular, the Renyi entropies in the vicinity of $\Delta=-1$ show signature of an essential singularity $[11,12]$. The purpose of this work is to understand how this change in the behavior of the system is reflected at the level of the ES.

The $\Delta=-1$ point is unitarily related to the $S U(2)$-invariant Heisenberg ferromagnetic chain, and hence the ground state is the degenerate $S U(2)$ multiplet corresponding to the highest total spin $S_{T}$. At $\Delta=-1$ the states of the multiplet are flat superpositions of all the possible spin configurations compatible with total magnetization, $S_{T}^{z}$, i.e., the particles are fully delocalized. We therefore refer to the ground state multiplet as the symmetric multiplet. In other terms at $\Delta=-1$ the ground state shows a "mean field" structure, i.e., the system has no notion of distance. This implies for example that there is no difference between open and periodic boundary conditions.

While there are well-known many-body model systems lacking notions of distance, such as the Lipkin-Meshkov-Glick model [14, 15, 16, 17] and the Richardson model [18], these are generally mean-field like at the level of the Hamiltonian. The specialty of the present case is that the XXZ interaction is local, and the symmetric structure appears only at a 
single value $(\Delta=-1)$ of the anisotropy parameter. Varying $\Delta$ is a natural way to tune away from the symmetric structure. In this Article, we show how this change from the symmetric to the more usual situation is manifested in the ES.

Although the usual interest is in entanglement properties of the ground state, in the vicinity of $\Delta=-1$ it is natural to study the entire set of states which become degenerate at that point. The ES properties we report are found to be common to the entire symmetric sector. For simplicity of demonstration or explicit calculations, we find it convenient to sometimes focus on simpler large- $S_{T}^{z}$ members of this manifold (which in the energy spectrum are near the top of this group of states for $\Delta>-1$ ) in addition to the $S_{T}^{z}=0$ ground state.

At $\Delta=-1$, the symmetric structure fully determines the $\operatorname{ES}[19,20,21,22]$. However, at $\Delta>-1$ the ES is not restricted, and in the limit $\Delta \rightarrow-1^{+}$most of the ES levels diverge (i.e. most of the eigenvalues of $\rho_{A}$ vanish). The divergent levels correspond to components in the ground state wavefunction at $\Delta>-1$ that do not reflect the symmetric structure present at $\Delta=-1$. Moreover, while at $\Delta=-1$ the ES does not contain any information about the boundary conditions or the geometry of the partitioning between $A$ and $B$, for $\Delta>-1$ the ES for periodic and open boundary conditions display striking differences. For open boundary conditions the ES is less dense (some of the ES levels are missing). This is related to there being two boundaries between $A$ and $B$ for periodic boundary conditions (and only one for open). This difference is also present in the behavior of the entanglement entropy in the gapless phase (as $1 / 3 \log \ell$ for periodic and as $1 / 6 \log \ell$ for open boundary conditions with $\ell$ the length of subsystem $A$ ). We also find that the multiplicity count of the ES in the vicinity of $\Delta=-1$ (numbers of ES levels diverging at different rates) seems to be described by an elegant combinatorial formula which we present as a conjecture.

We also analyze the entanglement eigenfunctions, i.e. the eigenstates of $\rho_{A}$. While the eigenfunctions corresponding to the lower (non-diverging) ES levels mimic the behavior of the ground state wavefunction (i.e. they exhibit a symmetric structure in the limit $\left.\Delta \rightarrow-1^{+}\right)$, the others show richer structures and in some cases their leading behavior (in $\epsilon \equiv \Delta+1$ ) can be given in terms of known polynomial functions.

Finally we investigate the distribution of the ES levels in the vicinity of the ferromagnetic point. Although in the thermodynamic limit this is expected to be a universal function, for finite chains the ferromagnetic point introduces significant corrections in the whole region $\Delta<0$.

For our analysis we take advantage of the fact that the XXZ is an integrable model and all its eigenstates can be in principle calculated using Bethe ansatz. We also use exact numerical diagonalization. Since ES levels are determined often up to values of $\sim 100$, the reduced density matrix eigenvalues $\lambda_{i}=e^{-\xi_{i}}$ need to be calculated with very high precision. Therefore, as in Ref. [4], we use arbitrary precision numerics.

In section 2 we discuss the spin- $\frac{1}{2} X X Z$ chain around $\Delta=-1$, showing the ground state manifold and how the wavefunctions of this symmetric sector behave at $\Delta \rightarrow-1^{+}$. In section 3 we present the main features of the ES, reviewing the exact structure known 
at $\Delta=-1$ and then presenting numerical diagonalization data for $\Delta=-1+\epsilon$. In section 4 we treat the ES perturbatively in the distance $\Delta+1$ from the ferromagnetic point, using the Bethe ansatz. The combinatorial aspects of the ES are also addressed and characterized. Section 5 is devoted to the study of the entanglement eigenfunctions. In section 6 we investigate how the distribution of the ES levels changes as the system is detuned away from the ferromagnetic point. Details of the Bethe ansatz calculation appear in the Appendix.

\section{The XXZ in the limit $\Delta \rightarrow-1^{+}$: the symmetric multiplet, energy spectrum and wavefunctions}

The spin- $\frac{1}{2} X X Z$ open chain of $L$ sites is defined by the Hamiltonian

$$
\mathcal{H}=\frac{1}{2} \sum_{i=1}^{L-1}\left(S_{i}^{+} S_{i+1}^{-}+S_{i}^{i} S_{i+1}^{+}\right)+\Delta \sum_{i=1}^{L-1} S_{i}^{z} S_{i+1}^{z}
$$

and the periodic chain is obtained by adding terms connecting sites $L$ and 1 . At $\Delta=-1$ the model is related to the ferromagnetic Heisenberg model $\left(-\sum_{i} \vec{S}_{i} \cdot \vec{S}_{i+1}\right)$ by the local unitary transformation $S^{x, y} \rightarrow-S^{x, y}$ applied on every second site of the chain $\ddagger$. The ground state of the Heisenberg ferromagnet is in the $S U(2)$ multiplet with highest total spin $S_{T}$ (symmetric multiplet), i.e. the one containing the fully polarized state $|F\rangle \equiv|\uparrow \uparrow \uparrow \cdots\rangle$. The $L+1$ states of the degenerate multiplet are obtained from $|F\rangle$ by successive applications of the lowering operator $S_{T}^{-} \equiv \sum_{i} S_{i}^{-}$. It is conventional to refer to $|F\rangle$ as the "vacuum" and the overturned spins as 'particles'. The states $\left(S_{T}^{-}\right)^{M}|F\rangle$ are equal weight superpositions of all the possible spin configurations with $M$ down spins (particles). The magnetization is $S_{T}^{z}=L / 2-M$ in terms of the particle content $M$; either $\left(S_{T}^{z}\right.$ or $\left.M\right)$ is a good quantum number for all $\Delta$ and labels the states of the multiplet.

In Figure 1 (a) we show the energies of the states which are connected to the symmetric multiplet at $\Delta=-1$, as a function of $\Delta+1$. The energy levels in the vicinity of $\Delta=-1$ are

$$
E=-\frac{L}{4}+\left[\frac{L}{4}-\frac{1}{L-1}\left(\frac{L}{2}-S_{T}^{z}\right)\left(\frac{L}{2}+S_{T}^{z}\right)\right](\Delta+1) .
$$

The ground state at $\Delta>-1$ is the one with $S_{T}^{z}=0$, while the other states of the multiplet are excited states. In Figure 1 (b) we plot the wavefunction of the eigenstate with two particles (which is part of the symmetric multiplet but not the ground state) showing wavefunction components versus the relative position $\left(x_{2}-x_{1}\right)$ of the two particles. While at the point $\Delta=-1$ the wavefunction is completely flat (the two particles are completely delocalized), for $\Delta>-1$ this property is progressively lost farther away from $\Delta=-1$.

$\ddagger$ Since this transformation is a product of local unitary transformations, it does not affect the entanglement spectrum. 


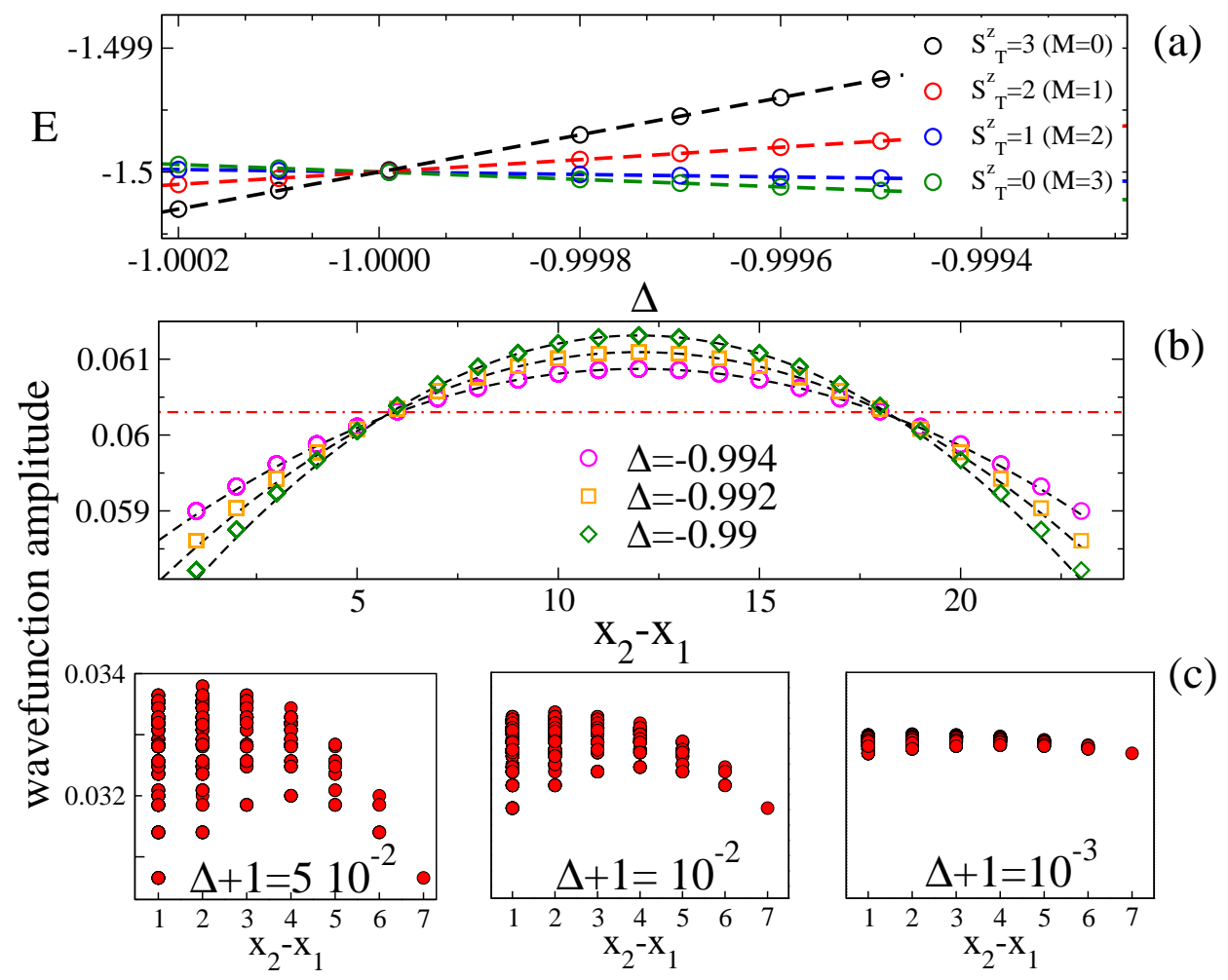

Figure 1. (a) Energy spectrum around $\Delta=-1$ of the XXZ spin chain with $L=6$ and periodic boundary conditions. We show the energies of all the chain eigenstates within the symmetric multiplet and $S_{T}^{z} \geq 0$. The energy at $\Delta=-1$ is $E=-L / 4$. The dashed lines are from Eq. (3). (b) The wavefunction of the state with only two particles $\left(M=2, S_{T}^{z}=L / 2-M\right)$ for a chain with $L=24$ and several values of $\Delta$. We plot the wavefunction components versus the interparticle distance $x_{2}-x_{1}$ $\left(x_{2}>x_{1}\right)$. The points are exact diagonalization data while the dashed line is the Bethe ansatz result, Eq. (A.4)). The dashed-dotted line is the asymptotic $(\Delta=-1)$ value, $\sqrt{2 /[L(L-1)]}$. (c) The wavefunction of the state with $M=L / 2(L=12)$ particles (ground state at $\Delta>-1$ ) plotted versus the relative distance of the first two particles. Data are obtained using Eq. (A.5). We show the wavefunction for three different values of $\Delta+1$, namely $\Delta+1=5 \cdot 10^{-2}, 10^{-2}, 10^{-3}$. At each fixed $x_{2}-x_{1}$ the different points correspond to the possible configurations of the other $L / 2-2$ particles.

This behavior is true for any state in the symmetric multiplet, although more complicated to display. For example in Fig. 1 (c) we show wavefunction components of the ground state $\left(S_{T}^{z}=0\right)$ for several values of $\Delta$. Now there are multiple components for each value of $x_{2}-x_{1}$. It is clear, however, that in the limit $\Delta \rightarrow-1$ the wavefunction becomes flatter and eventually becomes an equal weight superposition.

\section{Entanglement spectrum (ES) of the symmetric multiplet: general features}

In this section we describe how the structure of the ground state in the vicinity of $\Delta=-1$ determines the behavior of the ES. To calculate the ES we consider a bipartition of the 
chain into two parts $A$ and $B$ (of lengths $L_{A}$ and $L_{B}$ respectively). The wavefunction $|\psi\rangle$ of the chain, given two orthonormal bases for the subsystems $\left|\varphi_{i}^{A}\right\rangle,\left|\varphi_{j}^{B}\right\rangle$ can be written as

$$
|\psi\rangle=\sum_{i, j} \mathbf{M}_{i j}\left|\varphi_{i}^{A}\right\rangle \otimes\left|\varphi_{j}^{B}\right\rangle
$$

The matrix $\mathbf{M}_{i j}$ is a central object in the study of ES. The reduced density matrix eigenvalues $\lambda_{i}=e^{-\xi_{i}}$ [Eq. (1)] are the square of the Schmidt (singular) values of $\mathbf{M}$. Since the block magnetization $S_{A}^{z}$ is a good quantum number for the matrix $\mathbf{M}$ we can label the ES levels with $S_{A}^{z}$.

\subsection{The point $\Delta=-1$}

At $\Delta=-1$ the flat structure of the states in the symmetric sector, makes it possible to construct the matrix $\mathbf{M}$ explicitly and to calculate its singular values $\sqrt{\lambda_{i}}$. Any eigenstate in the symmetric multiplet is identified given the length of the chain $L$ and the magnetization $S_{T}^{z}$ (particle content). For each of these eigenstates there is one ES level per block magnetization sector $S_{A}^{z}$ which is given by [19]

$$
\xi_{\left\{S_{T}^{z}, S_{A}^{z}\right\}}^{\left\{L, L_{A}\right.}=-\log \left[\frac{\left(\begin{array}{c}
L_{A} \\
\frac{L_{A}}{2}-S_{A}^{z}
\end{array}\right)\left(\begin{array}{c}
L-L_{A} \\
\frac{L-L_{A}}{2}-S_{T}^{z}+S_{A}^{z}
\end{array}\right)}{\left(\begin{array}{c}
L \\
\frac{L}{2}-S_{T}^{z}
\end{array}\right)}\right]
$$

As a consequence of the delocalized structure of the states in the symmetric multiplet, Eq. (5) holds for both periodic and open boundary conditions.

After restricting to $S_{T}^{z}=0$, that is the ground state at $\Delta>-1$, and taking the thermodynamic limit $L \rightarrow \infty$ one obtains

$$
\xi_{\left\{S_{A}^{z}\right\}}^{\left\{L_{A}\right\}}=-\log \left[\frac{1}{2^{L_{A}}}\left(\begin{array}{c}
L_{A} \\
\frac{L_{A}}{2}-S_{A}^{z}
\end{array}\right)\right]
$$

which has also been obtained in [21].

\subsection{The ES in the limit $\Delta \rightarrow-1^{+}$: exact diagonalization results}

In Fig. 2 (a) we show the ES in the vicinity of $\Delta=-1$ (i.e. $\Delta+1 \equiv \epsilon=0$ ) for a block with $L_{A}=6$ in a chain with periodic boundary conditions and $L=18$ sites. For clarity we show only the sector of the ES with $S_{A}^{z}=0$, since the result in other $S_{A}^{z}$ sectors is qualitatively similar.

The ES levels show singular behavior. In the limit $\Delta \rightarrow-1^{+}$there is only one level converging to a finite value given by Eq. (5), while all the others diverge. The diverging behavior in the ES signals that in the limit $\Delta=-1^{+}$the wavefunction has to be a symmetric superposition of all allowed configurations. The components in the total wavefunction which reflect this symmetry give rise to a non diverging level, while the others contribute to the diverging ones. In Section 5 we will show how this distinction is 


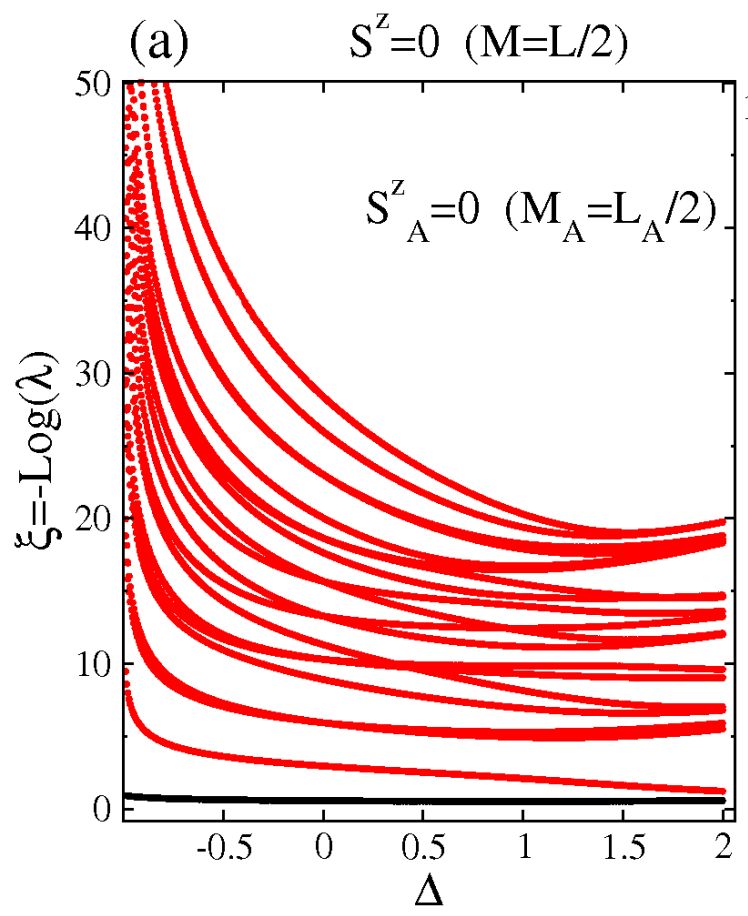

(b)

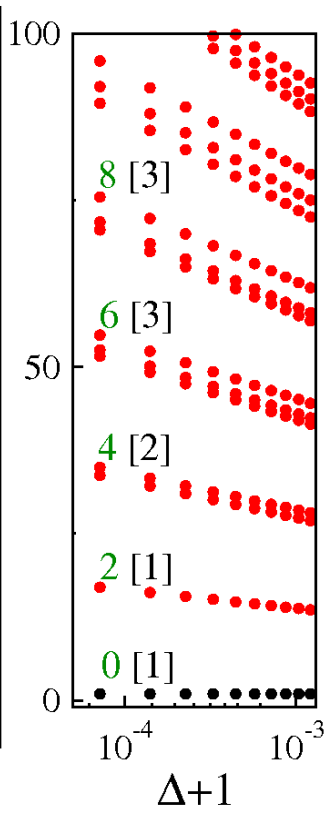

(c)

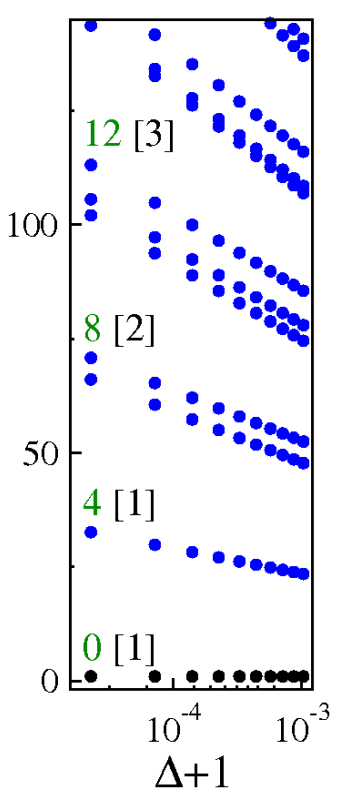

Figure 2. Entanglement spectrum from exact diagonalization $\left(L=18, L_{A}=6\right)$, plotted against $\Delta$. We show only the $S_{A}^{z}=0$ sector. In the limit $\Delta \rightarrow-1$ the lowest ES level (in black) converges to the finite value given by (5), while all the other levels diverge. (a) Periodic boundary conditions; wide range of $\Delta$. (b) Periodic boundary conditions; zooming around $\Delta=-1$. Log-scale on horizontal axis highlights the logarithmic divergence as $-\alpha \log (\Delta+1)+\beta$ of the levels. There are multiple ES levels for each value of $\alpha$. We use the notation $\alpha\left[m_{\text {per }}\right]$ where $m_{\text {per }}$ is the multiplicity of the set of levels with same exponent $\alpha$. (c) The same as in (b) but for a chain with open boundary conditions.

manifested in the structure of entanglement eigenfunctions corresponding to diverging and non-diverging ES levels.

In Fig. 2 (b) we demonstrate that the behavior of the levels in the vicinity of $\Delta=-1$ is given by $-\alpha \log (\Delta+1)+\beta, \alpha$ being an integer. The slopes $\alpha$, going from bottom to top in the ES, are given by the sequence $0,2,4,6, \ldots$ where 0 refers of course to the lowest level. A diverging level in the ES as $-\alpha \log (\Delta+1)$ corresponds to an eigenvalue of the reduced density matrix which vanishes as $(\Delta+1)^{\alpha}$. Focusing on the number of levels diverging with the same slope, an intriguing multiplicity sequence appears as higher levels in the ES are considered. In Fig. 2 (b) we use the notation $\alpha\left[m_{p e r}\right], m_{\text {per }}$ being the number of levels with slope $\alpha$. We observed that $m_{p e r}(\alpha)$ is given by the sequence $m_{\text {per }}(\alpha)=1,1,2,3, \ldots$..

In Fig. 2 (c) we show the same plot but for a chain with open boundary conditions $(L=17)$. The ES shows the same qualitative behavior, although now the slopes are $\alpha=0,4,8,12 \ldots$. The multiplicity structure apparently is described by the sequence $m_{\text {open }}(\alpha)=m_{\text {per }}(\alpha / 2)$. 

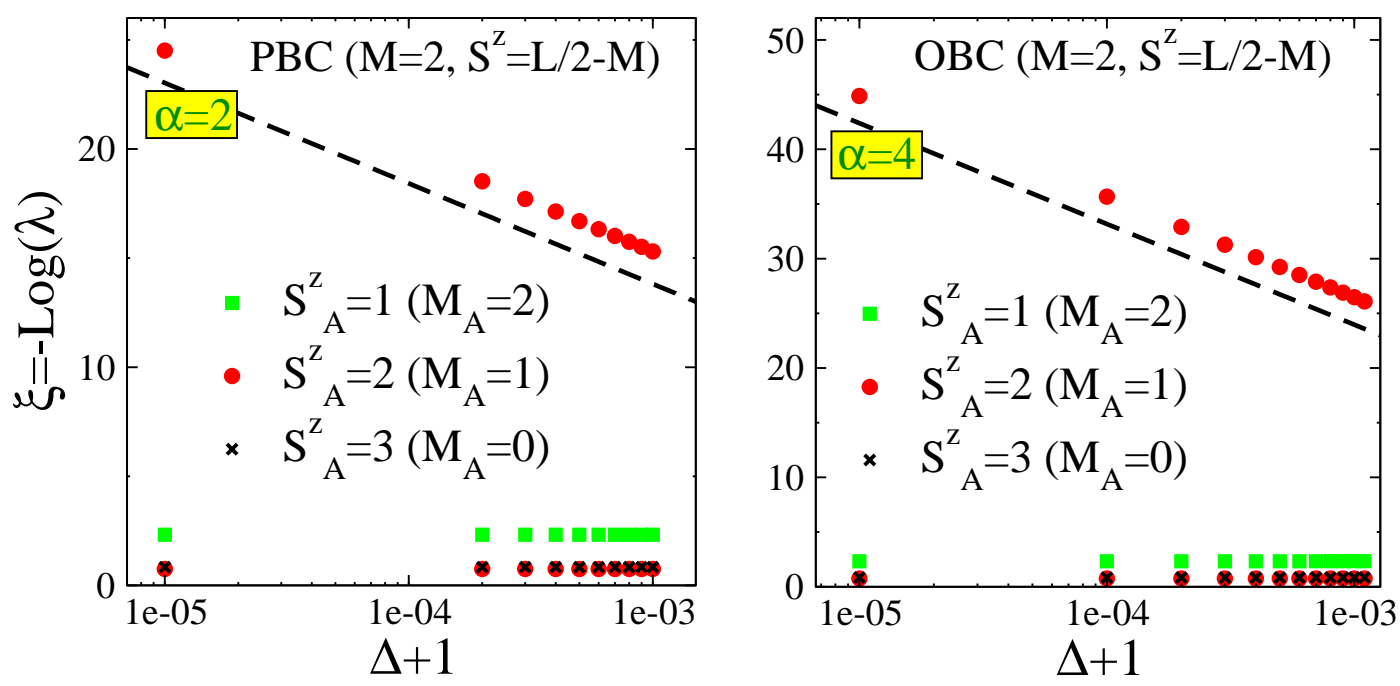

Figure 3. $\quad$ ES for the member of the symmetric multiplet containing only two particles $(M=2)$, plotted versus $\Delta+1$ for periodic (PBC) and open (OBC) boundary conditions. The block size is $L_{A}=6 . S_{A}^{z}\left(M_{A}\right)$ is the magnetization (particle content) of block $A$. The behavior of the diverging ES levels is $\xi=-\alpha \log (\Delta+1)+\beta$. The dashed lines are guides to the eye.

In a generic sector with $S_{A}^{z} \neq 0$, the slope sequence (the $\alpha$ 's) remains the same ( $\alpha=0,2,4, \ldots$ for periodic boundary conditions and $\alpha=0,4,8, \ldots$ for open boundary conditions), but the multiplicity structure is different.

The outlined scenario depends only on the symmetric structure of the states in the limit $\Delta \rightarrow-1^{+}$, thus the ES obtained from any state within the symmetric multiplet is expected to show similar features. This observation is supported in Fig. 3 where we show the full ES obtained from the state in the symmetric multiplet with only two particles (which is not the ground state for $\Delta>-1$, see Fig. 1). We show the ES for both periodic (left) and open boundary conditions (right). In both cases there are only three possible sectors $S_{A}^{z}$ determined by the number of ways of distributing the two particles in $A$ and $B$. Nonetheless, the main features observed in Fig. 2 are present: (a) for each value of $S_{A}^{z}$ there is one ES level converging to a finite value in the limit $\Delta \rightarrow-1^{+}$and this value is given by Eq. (5); (b) the slope of the only divergent level is 2 for periodic boundary conditions and 4 for open, in agreement with the sequences shown in Fig. 2.

\section{The ES at $\Delta \rightarrow-1^{+}$: perturbative and combinatorial structure}

The structure of the ES outlined in the previous sections can be quantitatively understood by expanding the state wavefunction for small $\epsilon \equiv \Delta+1$ and then calculating the ES. For the XXZ we can exploit the fact that the model is integrable and use the exact expression of the eigenstate wavefunctions (see Appendix A for details).

For this description, we choose the orthonormal basis $\left\{\left|\varphi_{i}^{A}\right\rangle\right\}=\left\{\left|x_{1}, x_{2}, \cdots, x_{M_{A}}\right\rangle\right\}$ 
for the $A$ block, where $\left|x_{1}, x_{2}, \cdots, x_{M_{A}}\right\rangle$ are the block configurations with particles at positions $x_{1}, x_{2}, \cdots, x_{M_{A}}$. Similarly for the $B$ subsystem we have $\left\{\left|\varphi_{i}^{B}\right\rangle\right\}=$ $\left\{\left|x_{M_{A}+1}, x_{M_{A}+2}, \cdots, x_{M}\right\rangle\right\}$.

With this notation the elements of the $\mathbf{M}$ matrix [Eq. (4)] are

$$
\mathbf{M}_{\left[x_{M_{A}+1}, \cdots, x_{M}\right]}^{\left[x_{1}, x_{2}, \cdots, x_{M_{A}}\right]} \equiv a\left(x_{1}, x_{2}, \cdots, x_{M_{A}}, x_{M_{A}+1}, \cdots, x_{M}\right)
$$

where $\left[x_{1}, x_{2}, \cdots, x_{M_{A}}\right]$ and $\left[x_{M_{A}+1}, \cdots, x_{M}\right]$ act as row or column indices. Here $a\left(x_{1}, x_{2}, \cdots, x_{M_{A}}, x_{M_{A}+1}, \cdots, x_{M}\right)$ is the amplitude of the chain configuration with particles at positions $x_{1}, x_{2}, \cdots, x_{M_{A}}, x_{M_{A}+1}, \cdots, x_{M}$. It turns out that the first order in $\Delta+1$ for the amplitude $a$ in (7) can be obtained analytically for arbitrary $M$ and system sizes $L$ and the resulting ES calculated.

In the three subsections below, we first treat the simplest nontrivial member of the symmetric multiplet (subsection 4.1), namely the one with only two particles $(M=2)$, and provide the first order expansion in $\epsilon \equiv \Delta+1$. In 4.2, we discuss $M>2$ and higher orders in $\epsilon$, providing a conjecture for the multiplicity sequence of the ES at different orders. In 4.3 we comment on the open-boundary case.

\subsection{The sector with two particles $(M=2)$ : first order in $\Delta+1$}

In the sector with two particles in the full chain wavefunction, using the expansion (A.4) and (7) the $\mathbf{M}$ matrix can be written explicitly. The possible values of $M_{A}$ are $M_{A}=0,1,2$. Moreover the blocks of $\mathbf{M}$ corresponding to $M_{A}=0,2$ are $1 \times 1$ (i.e. just numbers). In Fig. 3 (PBC) these would give the two ES levels with $S_{A}^{z}=1,3$, which are not diverging in the limit $\Delta \rightarrow-1^{+}$. For these two levels the first order contribution to the wavefunction gives a first order renormalization of the result (5). After restricting to the case $L_{A}=L_{B}=L / 2$ to simplify the expressions that would be otherwise very cumbersome, the ES level in the sector with $M_{A}=2$ is written as

$$
-2 \log \left[\frac{1}{2} \sqrt{\frac{L-2}{L-1}}\left(1-\frac{L(L-2)}{24(L-1)}(\Delta+1)\right)\right]
$$

Since we chose $L_{A}=L_{B}$ the ES level with $M_{A}=0$ is the same.

More interesting is the sector with $M_{A}=1\left(S_{A}^{z}=2\right.$ in Fig. 3), in which case there is a divergent level. The block in matrix $\mathbf{M}$ describing this sector reads

$\mathbf{M}=\sqrt{\frac{2}{L(L-1)}}\left[\left(1+A_{2} \epsilon\right) w^{(0)} \otimes w^{(0)}+\frac{\epsilon}{L-1}\left[w^{(0)} \otimes w^{(2)}+0 \leftrightarrow 2\right]-\frac{2 \epsilon}{L-1} w^{(1)} \otimes w^{(1)}\right](9)$

where $\otimes$ is the Kronecker product and we defined the three vectors $w_{k}^{(i)} \equiv k^{i}$ and $\epsilon \equiv \Delta+1$. The factor $A_{2}$ ensures the normalization of the full chain wavefunction (see Appendix A). M is a symmetric matrix and quadratic polynomial as a function of the coordinates of the two particles. Since the vectors $w^{(i)}$ are linearly independent, the rank of $\mathbf{M}$ is three. However, while two of the singular values of (9) are $\mathcal{O}(1)$ and $\mathcal{O}(\epsilon)$, the other one is $\mathcal{O}\left(\epsilon^{2}\right)$ and it is not meaningful because the wavefunction is accurate only up to first order. The third singular value is thus "fake" and the effective rank 
of (9) is two. (See Ref. [4] for a detailed discussion of such spurious eigenvalues in perturbative ES calculations.) One ES level of (9) is given by

$$
-2 \log \left[\frac{1}{24 \sqrt{2}} \frac{L^{2}(L+2)(L-2)}{(L(L-1))^{\frac{3}{2}}}(\Delta+1)\right]
$$

which diverges in the limit $\Delta \rightarrow-1$ and corresponds to the divergent ES level in Fig. 3 (PBC). The non diverging ES level in this sector up to first order is given by

$$
-2 \log \left[\sqrt{\frac{L^{2}}{2 L(L-1)}}+\frac{(L-2)[L(L-1)]^{\frac{3}{2}}}{12 \sqrt{2}(L-1)^{3}}(\Delta+1)\right] .
$$

Note that the correction to the singular values, at first order in $\epsilon \equiv(\Delta+1)$, is always of type $\sim L \epsilon$ for large $L$ [Eqs. (8),(10),(11)]. Thus $L \epsilon=L(\Delta+1)$ needs to be small for these expansions to be meaningful.

\subsection{Many particles $(M>2)$ and higher orders in $\Delta+1$ : combinatorial structure of} the ES

In this section we discuss how the structure of the ES for any state within the symmetric multiplet builds up as the particle number $(M)$ of the wavefunction increases and higher orders in $\Delta+1$ are taken into account.

In each $M_{A}$ sector the ES levels are organized in powers of $\Delta+1$ : the lowest ES level converges to a finite value in the limit $\Delta \rightarrow-1$, while the higher levels diverge as $-\alpha \log (\Delta+1)+\beta$ with $\alpha=2,4,6, \ldots$ as observed in Fig. 2.

Within a given $S_{A}^{z}$ sector and for each value of the slope $\alpha$ there is more than one level, i.e. a non trivial multiplicity structure arises as higher levels in the ES are considered.

It is natural to investigate how the multiplicity sequence depends on the particle content $(M)$. To this purpose in Fig. 4 we provide examples of multiplicity sequences in the ES for $M_{A}=2$ and $M=3,4,5$ (we consider a chain with $L=18$ and $L_{A}=8$ ). We note that the sequences can be obtained as particular cases from the expansion of the so called q-deformed binomial which is defined as:

$$
C_{q}(r, s)=\left(\begin{array}{l}
r \\
s
\end{array}\right)_{q} \equiv \frac{\prod_{k=r}^{r-s+1}\left(1-q^{k}\right)}{\prod_{k=1}^{r}\left(1-q^{k}\right)}
$$

For example, if we fix $M=r=5, M_{A}=s=2$ we obtain $C_{q}(5,2)=1+q+2 q^{2}+$ $2 q^{3}+2 q^{4}+q^{5}+q^{6}$. The sequence $1,1,2,2,2,1,1$ given by the coefficients of the different monomials matches the one shown in Fig. $4(\mathbf{c})$. It can be checked that $C_{q}(4,2), C_{q}(3,2)$ give the other sequences in Fig. 4. We also checked that the result holds for other values of $M, M_{A}$. Thus we conjecture that for all the states within the symmetric multiplet the multiplicity of the ES level diverging as $-\alpha \log (\Delta+1)$ is given by the coefficient of $q^{\alpha / 2}$ in the expansion of the q-deformed binomial (12) with $r=M, s=M_{A}$. This result 

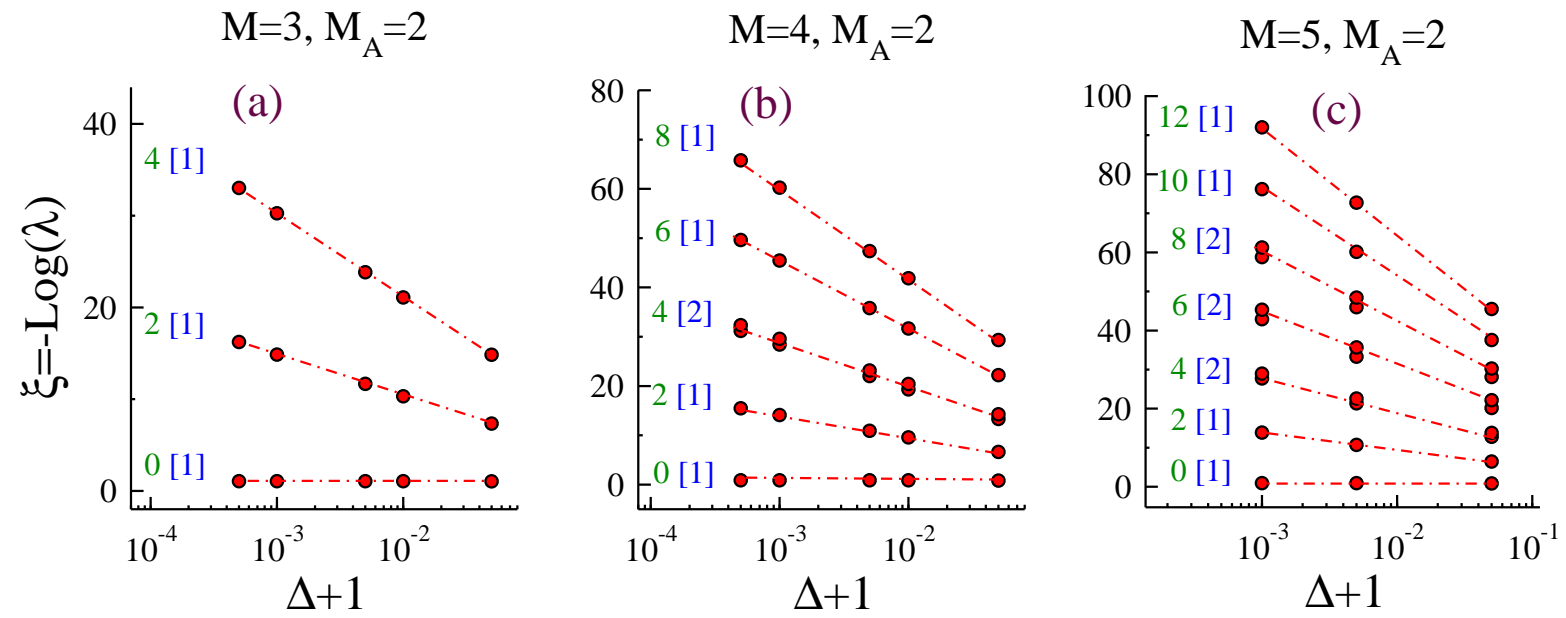

Figure 4. ES for a chain of $L=18$ sites, $L_{A}=8$, periodic boundary conditions and several values of $\Delta$ (arbitrary precision exact diagonalization data). $M\left(M_{A}\right)$ is the number of particles in the full chain ( $A$ block). Logarithmic scale on the horizontal axis highlights the divergent behavior $(\xi=-\alpha \log (\Delta+1)+\beta$ with $\alpha=0,2,4, \ldots)$. We use the same notation $\alpha\left[m_{\text {per }}\right]$ as in Fig. 2 for divergence exponent and multiplicity.

holds provided that $M<L_{A} \S$. We observe that for $L_{A} \leq M \leq L_{B}$ the multiplicity sequence is given by (12) with $r=L_{A}, s=M_{A}$, while if $M>L_{B}$ it is $r=L_{A}-\left(M-L_{B}\right)$ $s=M_{A}-\left(M-L_{B}\right)$.

\subsection{Open boundary conditions}

The XXZ chain with open boundary conditions can also be treated using the Bethe ansatz (details in Appendix A). The first order expansion of the matrix $\mathbf{M}$ is

$\mathbf{M}_{\left[x_{M_{A}+1}, \cdots, x_{M}\right]}^{\left[x_{1}, x_{2}, \cdots, x_{M^{\prime}}\right]}=\mathbf{1}+\epsilon\left[\mathcal{M}_{1}\left(x_{1}, x_{2}, \cdots, x_{M_{A}}\right)+\mathcal{M}_{2}\left(x_{M_{A}+1}, \cdots, x_{M}\right)\right]$

where $\mathcal{M}_{1}$ and $\mathcal{M}_{2}$ are polynomial functions that can be obtained from (A.8). We stress that (13) does not contain any cross terms of the form $x_{i} x_{j}$ with $x_{i} \in A$ and $x_{j} \in B$ (note the difference with the result (9) for periodic boundary conditions). This means that at first order in $\epsilon$ there is no entanglement between $A$ and $B$.

Despite the different values of $\alpha$ the multiplicity structure in the ES shows similarities with the case of periodic boundary conditions. In Fig. 5 we show the ES levels in the sector with $M_{A}=2$ and total number of particles $M=3,4,5$. As already observed in Fig. 2 all the ES levels with $\alpha=2 k$ and $k$ odd are missing. Nonetheless the multiplicity sequence $m_{\text {open }}(\alpha)$ is simply related to the one observed for periodic boundary conditions as $m_{\text {open }}(\alpha)=m_{\text {per }}(\alpha / 2)$ with $m_{\text {per }}$ given in terms of (12).

$\S$ Here we are considering the case $L_{A} \leq L_{B}$ otherwise the condition is $M<\min \left(L_{A}, L_{B}\right)$. However, since the reduced density matrices of the two subsystems are isospectral we can always choose subsystem $A$ such that $L_{A} \leq L_{B}$. 

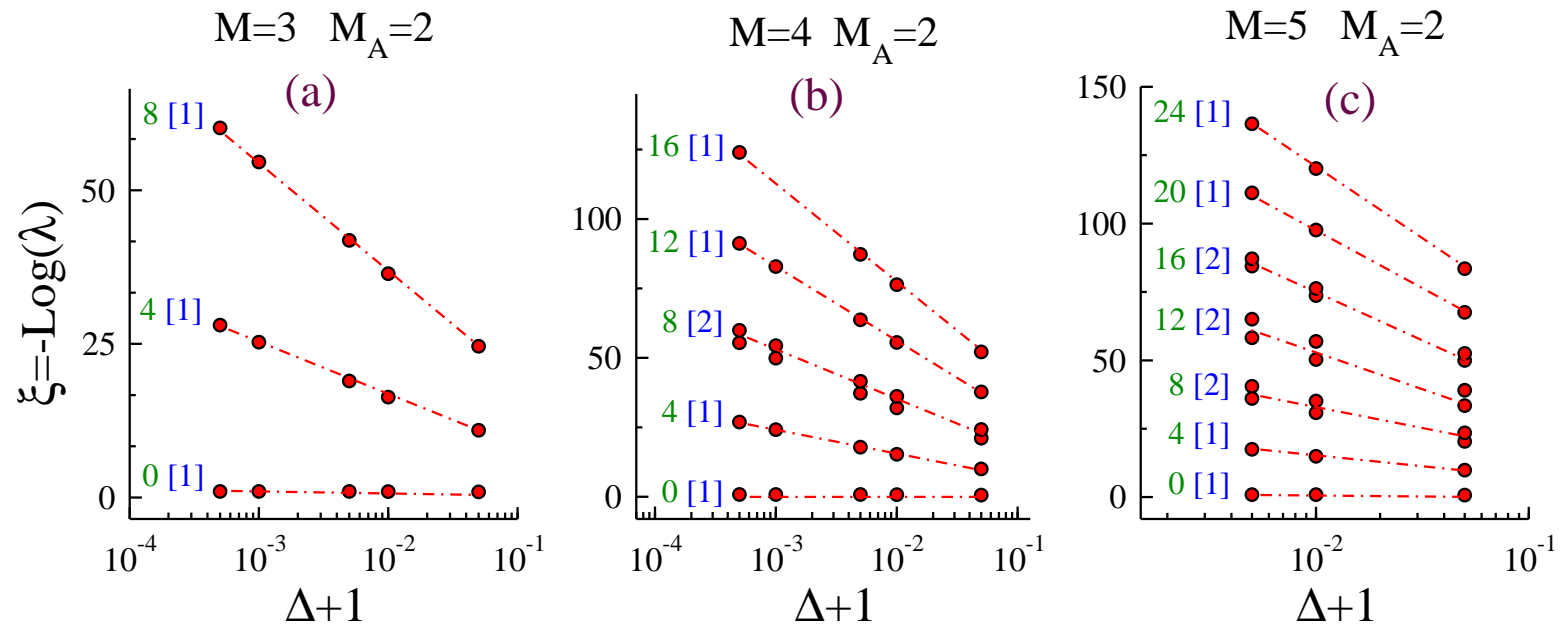

Figure 5. ES for a chain of $L=18$ sites, $L_{A}=8$, periodic boundary conditions and several values of $\Delta$ (arbitrary precision exact diagonalization data). $M\left(M_{A}\right)$ is the number of particles in the full chain ( $A$ block). Logarithmic scale on the horizontal axis highlights the divergent behavior $(\xi=-\alpha \log (\Delta+1)+\beta$ with $\alpha=0,4,8, \ldots)$. We use the same notation $\alpha\left[m_{\text {per }}\right]$ as in previous figures 2 and 4 for divergence exponent and multiplicity.

\section{The entanglement eigenfunctions at $\Delta \rightarrow-1^{+}$}

In this section we examine the entanglement eigenfunctions (eigenvectors of reduced density matrix). We show contrasting behavior for the eigenfunctions corresponding to the ES levels which diverge in the $\Delta \rightarrow-1^{+}$limit and those that do not diverge.

We discuss the case of a chain with periodic boundary conditions. We provide information about structure of the entanglement eigenfunctions in two ways. First, up to first order in $\Delta+1$ the Bethe ansatz is tractable for calculating the entanglement eigenfunctions for arbitrary $M, M_{A}, L$ (Section 5.1). Second, in the $M_{A}=1$ sector, numerical data reveals a polynomial sequence for the forms of the wavefunctions, which we express in terms of the discrete Chebyshev polynomials (Section 5.2).

\subsection{First order in $\Delta+1$}

We start discussing the sector with $M=2$. In this sector the entanglement eigenfunctions reflect the polynomial structure of the matrix $\mathbf{M}$ (see (9)).

In Fig. 6 (a) (b) we show the ES eigenfunctions for $M=2$ and $M_{A}=1$ obtained from the expansion (9). The lowest ES level eigenfunction ((a) in Fig. 6) has a parabolic dependence on $x_{1}$ (the position of the particle inside block $A$ ) and as the ferromagnetic point is approached it becomes flat. The non-diverging ES levels thus mimic the structure of the full chain eigenstate. The same behavior is present in the sectors with $M_{A}=0,2$, where the ES has only one (non diverging) level. In contrast, the eigenfunction of the first diverging ES level shows linear behavior as a function of $x_{1}$ (Fig. $6(\mathbf{b})$ ). 


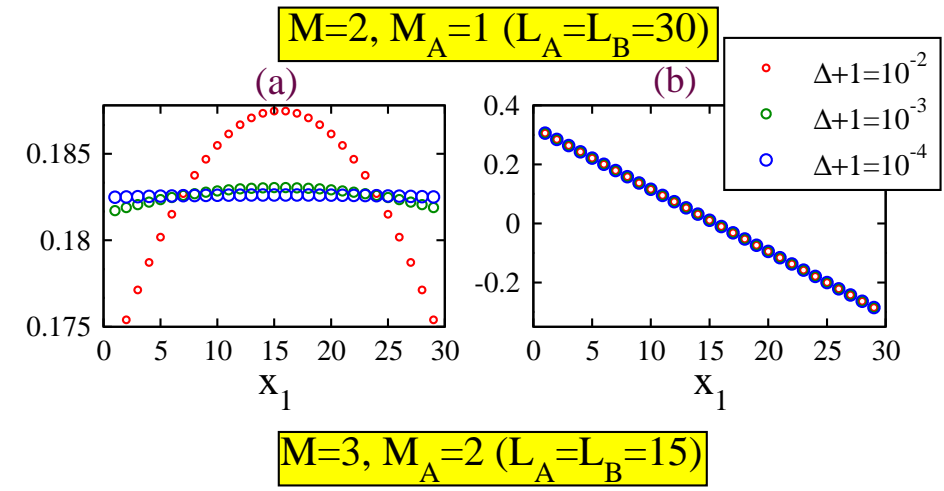

(c)

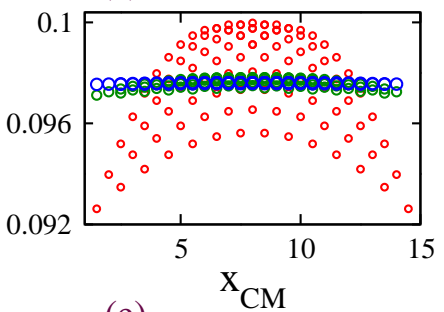

(e)

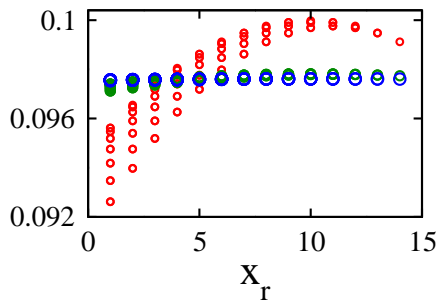

(d)
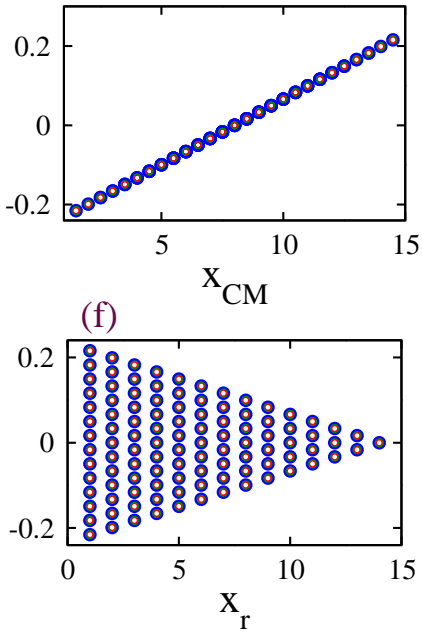

Figure 6. (a,b) Entanglement eigenfunctions in the sector with only one particle in the block $A\left(M_{A}=1\right)$ and two particles in the full chain wavefunction $(M=2)$ at different values of $\Delta$. On the x-axis $x_{1}$ is the position of the particle inside block $A$. (a) is the eigenfunction corresponding to the lowest non-diverging ES level while in (b) we show the eigenfunction for the first diverging ES level (see Fig. 3). $(\mathbf{c}, \mathbf{d}, \mathbf{e}, \mathbf{f})$ Entanglement eigenfunctions in the sector with two particles in the block $\left(M_{A}=2\right)$. In $(\mathbf{c})(\mathbf{e})$ we show the entanglement eigenfunction for the lowest ES level versus $x_{C M} \equiv\left(x_{1}+x_{2}\right) / 2$ and $x_{r} \equiv x_{2}-x_{1}$ where $x_{1} x_{2}$ are the position of the two particles. In (d)(f) we show the entanglement eigenfunction of the first divergent ES level.

In Fig. 6 (c)-(f) we show the entanglement eigenfunctions for the case with two particles in the block $A\left(M_{A}=2\right)$ and only one in $B(M=3)$. In $(\mathbf{c})$ and $(\mathbf{d})$ we plot the eigenfunction components versus the "center of mass" coordinate $\left(x_{1}+x_{2}\right) / 2$ of the two particles in $A$. The entanglement eigenfunction of the lowest ES level (c) exhibits the flattening behavior characteristic of the chain wavefunction, while the eigenfunction in (d) is again linear. The same qualitative behavior is observed in the relative distance $x_{2}-x_{1}$ (see Fig. 6 (e) (f)).

This result is general for all $M_{A}, M$ sectors - the entanglement eigenfunctions for the non-diverging ES levels flatten in the limit $\Delta \rightarrow-1^{+}$, while the eigenfunctions for the ES diverging as $-\log (\Delta+1)$ are linear functions of the coordinates $x_{1}, x_{2}, \ldots, x_{M_{A}}$. 

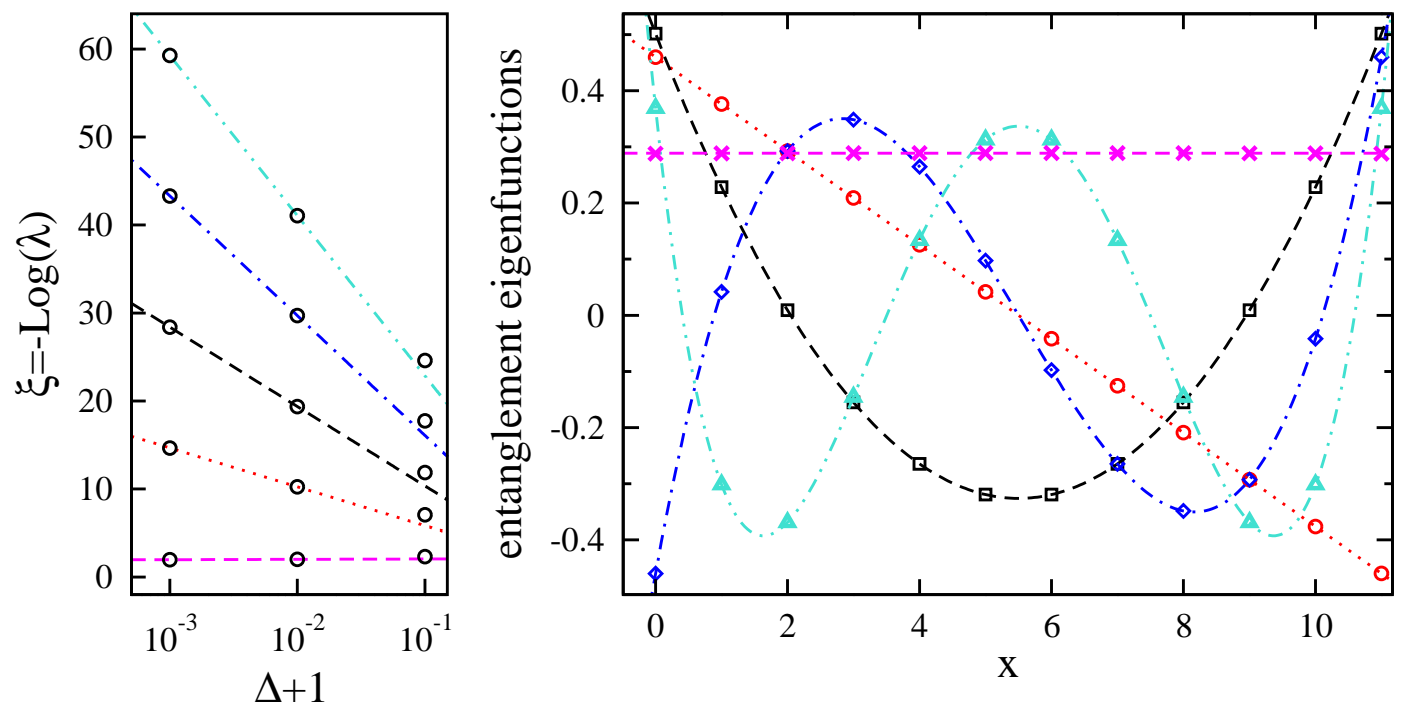

Figure 7. ES and entanglement eigenfunctions in the sector with $M_{A}=1$ (exact diagonalization data). (left) The ES versus $\Delta+1$ for a block with $L_{A}=12$ embedded in a chain of size $L=24$. We show the ES levels in the sector with only one particle in the block $\left(M_{A}=1\right)$ and five particles in the full chain $(M=5)$. The lines are fits to $-\alpha \log (\Delta+1)+\beta$. (right) For each level in the ES we show with the same color the components in the corresponding entanglement eigenfunction for $\Delta+1=10^{-3}$. Here $x$ is the position of the particle in block $A$. The lines are the polynomial behaviors given by Eq. (16).

\subsection{Entanglement eigenfunctions in the sector with $M_{A}=1$ and arbitrary $M$}

In the $M_{A}=1$ sector the number of ES levels is given by the total number of particles $M$ [Eq. 12]. These levels are shown in Fig. 7 (left) for $M_{A}=1$ and $M=5$. For each of the ES levels we report (right) the components of the corresponding entanglement eigenfunction versus the position of the particle in block $A$.

We observe that in the limit $\Delta=-1^{+}$the entanglement eigenfunction of the level appearing at order $n$ (i.e. diverging as $-\alpha \log (\Delta+1)$ with $\alpha=2 n$ ) is a polynomial of order $n$ (as a function of the position of the particle in block $A$ ). This is the only information needed in order to know the exact functional form of the entanglement eigenfunctions. The polynomial can be fixed by imposing that the eigenfunctions are mutually orthogonal and are normalized.

For instance the entanglement eigenfunction of the lowest ES level is the flat superposition $\left|\varphi_{0}\right\rangle=\sum_{x} c|x\rangle$, with $|x\rangle$ the configuration with the particle at position $x$ in block $A$. The coefficient $c$ is determined by imposing the normalization of the eigenfunction. For the first diverging ES level in Fig. 7 (the red-dotted line) one has $\left|\varphi_{1}\right\rangle=\sum_{x}\left(c^{0}+c^{1} x\right)|x\rangle$. The coefficients $c^{0}, c^{1}$ are fixed by imposing that $\left\langle\varphi_{0} \mid \varphi_{1}\right\rangle=0$ and that $\left|\varphi_{1}\right\rangle$ is normalized. This gives

$$
\left|\varphi_{1}\right\rangle=\sqrt{\frac{12}{L_{A}\left(L_{A}^{2}-1\right)}} \sum_{x}\left(x-\frac{L_{A}-1}{2}\right)|x\rangle
$$


The next diverging ES level (the black-dashed line) is instead

$$
\left|\varphi_{2}\right\rangle=\sqrt{\frac{5}{L_{A}\left(L_{A}^{4}-5 L_{A}^{2}+4\right)}} \times \sum_{x}\left(2+L_{A}^{2}+3 L_{A}+6 x^{2}-6\left(L_{A}-1\right) x\right)|x\rangle(15)
$$

The procedure allows to obtain the leading order of all the entanglement eigenfunctions in the sector $M_{A}=1$. The set of polynomials obtained are known as the discrete Chebyshev polynomials $T_{n}[23]$ and are defined as

$$
T_{n}(x)=n ! \mathcal{D}^{n}\left(\begin{array}{l}
x \\
n
\end{array}\right)\left(\begin{array}{c}
x-L_{A} \\
n
\end{array}\right)
$$

where $\mathcal{D}$ is such that $\mathcal{D} f(x) \equiv f(x+1)-f(x)$.

6. Away from $\Delta=-1$ : the ES distribution in the gapless phase $(\Delta>-1)$

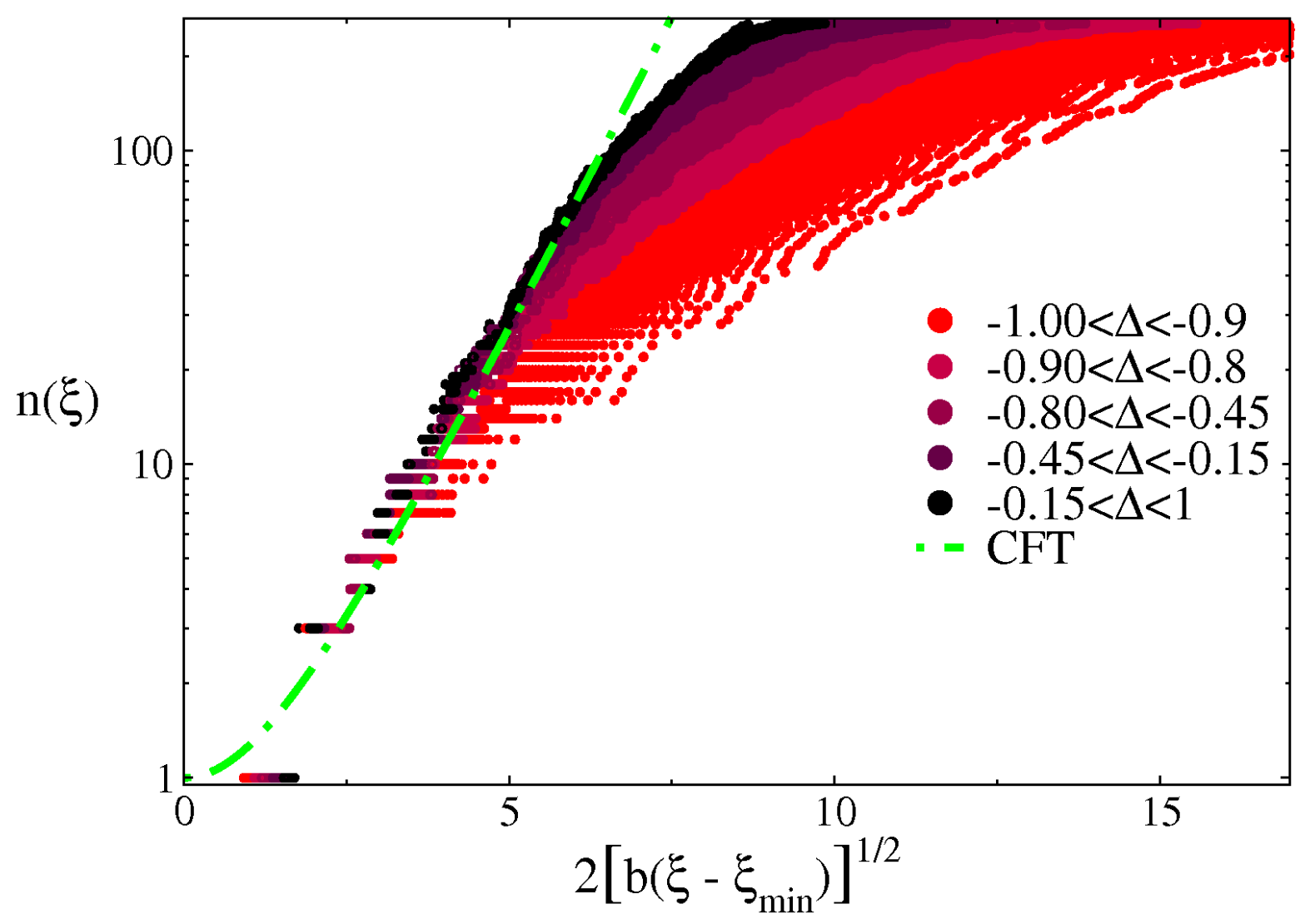

Figure 8. Distribution of the ES for a block with $L_{A}=8$ embedded in a chain of $L=18(M=9)$ sites and several values of $\Delta$ (exact diagonalization). We denote with $n(\xi)$ the number of ES levels smaller than $\xi$. The line is the asymptotic CFT result (17).

In this section we show how the symmetric structure of the $\Delta=-1$ point affects the distribution of ES levels for $\Delta>-1$. We use periodic boundary conditions and consider 
$M=L / 2$ (i.e. the state in the multiplet that is the ground state at $\Delta>-1$ ). It has been shown [10] that for 1D gapless systems described by a conformal field theory (CFT) the asymptotic distribution of the ES levels is given by

$$
n(\xi)=I_{0}\left(2 \sqrt{b\left(\xi-\xi_{\text {min }}\right)}\right)
$$

where $n(\xi)$ is the number of ES levels smaller than $\xi, \xi_{\text {min }}$ is the value of the lowest ES level, $b=c / 6 \log \left[L \sin \left(\pi / L L_{A}\right)\right], c$ is the central charge $(c=1$ for the $X X Z$ spin chain) and $I_{0}$ the modified Bessel function of order zero. This ES distribution depends only on the central charge (in this sense is super-universal), i.e. (17) holds irrespectively of the value of $\Delta$ in the gapless phase.

However, the ferromagnetic point $\Delta=-1$ (where the model is not conformal invariant) is the endpoint of the $c=1$ conformal line which describes the $X X Z$ at $-1<\Delta \leq 1$. Although in the thermodynamic limit (when all the length scales are sent to infinity) the $C F T$ result is expected to describe the ES distribution in the whole region $-1<\Delta \leq 1$, for a finite chain deviations from the CFT prediction are expected especially in the vicinity of $\Delta=-1$. At the $\Delta=-1$ point itself, the von Neumann and the Renyi entropies show scale invariance but not conformal invariance [21]. The asymptotic behaviors are [21]

$S_{A}^{(\gamma)}=\frac{1}{2} \log \left(\frac{\pi L_{A}}{2}\right)+\frac{\log (\gamma)}{2(\gamma-1)}+\mathcal{O}\left(1 / L_{A}\right), \quad S_{A}=\frac{1}{2} \log \left(\frac{\pi L_{A}}{2}\right)+\frac{1}{2}+\mathcal{O}\left(1 / L_{A}\right)$

Although the scaling is logarithmic, the prefactors differ from the CFT prediction that would give $S_{A}^{(\gamma)}=\frac{c}{6}\left(1+\frac{1}{\gamma}\right) \log L_{A}+\mathcal{O}(1)$ and $S_{A}=\frac{c}{3} \log L_{A}+\mathcal{O}(1)$.

It is interesting to check how this is reflected at the level of the ES distribution. In Fig. 8 we show the ES distribution for a chain of $L=18$ and block size $L_{A}=8$. Finite size effects are present, but we still notice a good agreement between the numerical data and the CFT prediction (17) for $\Delta>-0.15$. The numerical data in the range $-0.15<\Delta<1$ collapse on the same curve, which is well described by the $C F T$ result. However, in the region $\Delta<-0.15$ no data collapse is observed and the $C F T$ prediction is a quite poor approximation. Thus for these block sizes and system sizes the ferromagnetic point $\Delta=-1$ appears to dominate the physics of the whole region $\Delta<0$.

\section{Conclusions}

In this paper we studied the ES of the spin- $\frac{1}{2} X X Z$ model in the limit $\Delta \rightarrow-1^{+}$ (ferromagnetic point) for a finite chain with periodic and open boundary conditions. To this purpose we used exact diagonalization (with arbitrary precision numerics) and Bethe ansatz. We have characterized the ES for all the chain eigenstates within the highest spin multiplet (symmetric multiplet) in the limit $\Delta \rightarrow-1^{+}$.

At $\Delta=-1$ these states are flat superpositions of all the spin configurations compatible with the state magnetization $S_{T}^{z}$. In other terms they show a "mean field" structure 
with no notion of distance. As a consequence the ES at $\Delta=-1$ for each of these eigenstates can be calculated exactly and shows few levels. At $\Delta>-1$ the "mean field" structure is progressively lost as we move away from the ferromagnetic point.

We summarize our results as follows:

(i) In the limit $\Delta \rightarrow-1^{+}$, most of the ES levels diverge. For each value of the block magnetization $S_{A}^{z}$ there is only one non diverging level which reflects components of the state wavefunction that in the limit $\Delta \rightarrow-1^{+}$are flat superposition of all the allowed spin configurations. The divergent behavior is given by $\xi=$ $-\alpha \log (\Delta+1)+\beta$ with $\alpha=0,2,4, \ldots$ for periodic boundary conditions and $\alpha=0,4,8, \ldots$ for open.

(ii) The multiplicity of an ES level diverging with slope $\alpha$ is given by the sequences $m_{\text {per }}(\alpha)$ for periodic and $m_{\text {open }}(\alpha)=m_{\text {per }}(\alpha / 2)$ for open boundary conditions. We have provided a combinatorial expression [Eq. (12)] for these sequences.

(iii) We have studied the entanglement eigenfunctions, i.e., the eigenfunctions of the reduced density matrix $\rho_{A}$. The eigenfunctions of the non diverging ES levels mimic the behavior of the state wavefunction, i.e., in the limit $\Delta \rightarrow-1^{+}$are flat superpositions of all the possible spin configurations. In contrast, the entanglement eigenfunctions of the diverging levels do not become flat but display rich structures. In particular, in the sector $M_{A}=1$ their leading order in $\Delta+1$ can be given analytically in terms of the discrete Chebyshev polynomials.

(iv) Although in the thermodynamic limit in the whole gapless phase at $-1<\Delta \leq 1$ the ES distribution is described by (17), for a finite chain significant deviations from the CFT prediction and non universal behavior are observed in the vicinity of $\Delta=-1$. Within the range of system sizes considered $\left(L_{A}=8\right)$ the ferromagnetic point $\Delta=-1$ appears to dominate the whole region $\Delta<0$. 


\section{Appendix A. Expansion of exact Bethe ansatz solutions around $\Delta=-1$}

In this Appendix we outline the Bethe ansatz expansions used in the Article.

\section{Appendix A.1. Periodic boundary conditions}

In the region $\Delta>-1$ the Bethe equations can be written in the form

$$
\arctan \left(\frac{v_{l}}{\tan (\zeta / 2)}\right)=-\frac{\pi}{L} I_{l}+\frac{1}{L} \sum_{m \neq l}^{M} \arctan \left(\frac{v_{l}-v_{m}}{\left(1-v_{l} v_{m}\right) \tan \zeta}\right)
$$

where $M$ is the number of particles in the chain, $v_{l}(l=1,2, \ldots, M)$ are the so called rapidities and $\zeta=\arccos \Delta . I_{l}$ are the Bethe numbers and each choice of $I_{l}$ identifies

an eigenstate of the $X X Z$ Hamiltonian. In each $S_{T}^{z}$ sector the lowest energy eigenstate (which is the state in the symmetric multiplet with magnetization $S_{T}^{z}$ ) is identified by the following choice of the Bethe numbers

$$
I_{l}=-(M+1) / 2+l \quad l=1,2, \ldots, M
$$

(Note $M=L / 2-S_{T}^{z}$.) From the solutions of the Bethe equations the state can be constructed as

$$
|\psi\rangle=\sum_{\mathcal{P}} \underbrace{(-1)^{\epsilon_{\mathcal{P}}} e^{\sum_{l} k_{\mathcal{P}_{l}} x_{l}+\frac{i}{2} \sum_{l<m} \Phi\left(k_{\mathcal{P}_{l}}, k_{\mathcal{P}_{m}}\right)}}_{a\left(x_{1}, x_{2}, \ldots, x_{M}\right)}\left|x_{1}, x_{2}, \ldots, x_{M}\right\rangle
$$

where $\mathcal{P}$ runs over all permutations of the set $\left\{x_{1}, x_{2}, \ldots, x_{M}\right\}, x_{l}$ being the particle positions. We denote with $\left|x_{1}, x_{2}, \ldots, x_{M}\right\rangle$ the spin configuration with particles (down spins) at sites $x_{1}, x_{2}, \ldots$. The parity of the permutation $\mathcal{P}$ is denoted by $\epsilon_{\mathcal{P}}$. We also defined $k_{l} \equiv \pi+2 \arctan \left(v_{l} / \tan (\zeta / 2)\right) \quad(\bmod 2 \pi)$ and the scattering phase $\Phi\left(k_{l}, k_{m}\right) \equiv$ $2 \arctan \left(\left(v_{l}-v_{m}\right) /\left(\left(1-v_{l} v_{m}\right) \tan \zeta\right)\right)$.

The idea of the approach is to solve the Bethe equations (A.1) perturbatively order by order in $\epsilon \equiv \Delta+1$ imposing a solution of the form

$$
v_{l}=v_{l}^{(0)}+v_{l}^{(1)} \epsilon+\ldots
$$

to obtain a perturbed expression for the state using (A.3). At zeroth order the Bethe equations are given by the set of equations

$$
v_{l}^{(0)}=\frac{2}{L} \sum_{m \neq l} \frac{1-v_{l}^{(0)} v_{m}^{(0)}}{v_{l}^{(0)}-v_{m}^{(0)}}
$$

Appendix A.1.1. First order expansion of the wavefunctions Up to first order in $\epsilon$ the amplitude (see (A.3)) of the wavefunction with two particles can be written analytically

$$
a\left(x_{1}, x_{2}\right)=\sqrt{\frac{2}{L(L-1)}}\left[1+A_{2} \epsilon-\frac{\epsilon}{L-1}\left(x_{1}-x_{2}+\frac{L}{2}\right)^{2}\right]
$$


where

$$
A_{2}=\frac{2}{L(L-1)^{2}} \sum_{x_{1}<x_{2}}\left(x_{1}-x_{2}+L / 2\right)^{2}
$$

ensures the correct first order normalization. The last term in (A.4) shows a quadratic behavior in the inter particle distance $x_{2}-x_{1}$ and linear dependence on $\epsilon$ of the "curvature" of the wavefunction. Eq. (A.4) also shows that the asymptotic limit $\epsilon \rightarrow 0$ the wavefunction becomes an equal weight superposition (no dependence on $x_{i}$ ). These features were shown and discussed in Figure 1, where we also compared with exact diagonalization data.

In first order it is possible to go beyond $M=2$ and derive the amplitudes for any state within the symmetric multiplet:

$$
a\left(x_{1}, x_{2}, \ldots, x_{M}\right)=\frac{1}{\sqrt{\mathcal{I}}}\left[1+A_{M} \epsilon-\frac{\epsilon}{L-1} \sum_{i<j}^{M}\left(X_{i j}+\frac{L}{2}\right)^{2}\right]
$$

where $\mathcal{I} \equiv 1 / M ! \prod_{k=0}^{M-1}(L-k), X_{i j} \equiv x_{i}-x_{j}$, and the normalization is

$$
A_{M}=\frac{1}{(L-1) \mathcal{I}} \sum_{x_{1}<x_{2}<\cdots<x_{M}} \sum_{i<j}\left(X_{i j}+\frac{L}{2}\right)^{2}
$$

While the $M=2$ wavefunction corresponds to an excited state at $\Delta>-1$, the formula above includes as a particular case $(M=L / 2)$ the wavefunction of the ground state in the gapless phase. One important property of (A.5) is that it is quadratic and symmetric in all the interparticle distances $x_{i}-x_{j}$. An example of wavefunction amplitudes obtained from (A.5) is shown in Fig. 1 (c).

\section{Appendix A.2. Open boundary conditions}

It is possible to treat the open boundary conditions case within the Bethe ansatz formalism [24]. The Bethe equations now read

$k\left(v_{l}\right)=\frac{\pi}{L+1} I_{l}-\frac{1}{L+1} \Phi\left(v_{l},-v_{l}\right)-\frac{1}{2} \frac{1}{L+1} \sum_{m \neq l}\left[\Phi\left(v_{l},-v_{m}\right)+\Phi\left(v_{l}, v_{m}\right)\right]$

with $\Phi\left(v_{l}, v_{m}\right) \equiv 2 \arctan \left(\left(v_{l}-v_{m}\right) /\left(\left(1-v_{l} v_{m}\right) \tan \zeta\right)\right)$ and $k\left(v_{l}\right) \equiv \pi+$ $2 \arctan \left(v_{l} \tan (\zeta / 2)\right)$. The Bethe numbers giving the lowest energy state in each magnetization sector (i.e. the states within the symmetric multiplet) are now $I_{l}=$ $\{1,2, \ldots, M\}$. The main difference with respect to the periodic boundary conditions is the presence of the "reflected" phase shifts $\Phi\left(v_{l},-v_{m}\right)$.

This fact has dramatic consequences in the wavefunction and therefore in the ES. The general expression of the chain wavefunction has the form

$$
|\psi\rangle=\sum_{\mathcal{P}}(-1)^{\epsilon_{\mathcal{P}}} A\left(v_{\mathcal{P}_{1}}, v_{\mathcal{P}_{2}}, \ldots, v_{\mathcal{P}_{M}}\right) e^{i\left(k_{\mathcal{P}_{1}} x_{1}+k_{\mathcal{P}_{2}} x_{2}+\cdots+k_{\mathcal{P}_{M}} x_{M}\right)}\left|x_{1}, x_{2}, \cdots, x_{M}\right\rangle
$$


where the sum is over all permutations and (arbitrary number of) reflections of the set of rapidities $\left\{v_{1}, v_{2}, \cdots, v_{M}\right\}$. The amplitude $A\left(v_{\mathcal{P}_{1}}, v_{\mathcal{P}_{2}}, \ldots, v_{\mathcal{P}_{M}}\right)$ is not as simple as for periodic boundary and we do not show it. As for periodic boundary conditions we can expand the Bethe equations in the vicinity of $\Delta=-1$. Quite interestingly now we find that the lowest order solutions are determined by the equations

$$
\frac{1}{v_{l}^{(0)}}=\frac{1}{L+1} \frac{1+\left(v_{l}^{(0)}\right)^{2}}{v_{l}^{(0)}}+\frac{1}{L+1} \sum_{m \neq l}\left[\frac{1-v_{l}^{(0)} v_{m}^{(0)}}{v_{l}^{(0)}-v_{m}^{(0)}}+\frac{1+v_{l}^{(0)} v_{m}^{(0)}}{v_{l}^{(0)}+v_{m}^{(0)}}\right]
$$

By expanding (A.8) in $\Delta+1$ we find quite remarkably that the sum over the reflections of the rapidities $v_{l} \rightarrow-v_{l}$ cancel at first order in $\Delta+1$ the products of the form $x_{i} x_{j}$. This fact leads to the suppression of the first order ES levels for open boundary conditions, as we have seen (Figures 2 and 5).We provide as an example of this cancellation the first order amplitude $a\left(x_{1}, x_{2}\right)$ for the (unnormalized) chain wavefunction in the case with two particles, which reads

$a\left(x_{1}, x_{2}\right)=1-\frac{\epsilon}{L}\left[L^{2} / 2+3-L\left(1 / 6+x_{1}+3 x_{2}\right)+2 x_{1}\left(x_{1}+1\right)+2 x_{2}\left(x_{2}+1\right)\right]$

Our numerical results indicate that the same type of cancellation will happen at every odd order, but for higher orders this is too cumbersome to perform explicitly.

\section{References}

[1] L. Amico, R. Fazio, A. Osterloh, and V. Vedral, Rev. Mod. Phys. 80, 517 (2008).

[2] I. Peschel, and V. Eisler, J. Phys. A: Math. Theor. 42, 504003 (2009). M.-C. Chung, and I. Peschel, Phys. Rev. B 64, 064412 (2001).

[3] H. Li and F. D. M. Haldane, Phys. Rev. Lett. 101, 010504 (2008). A. M. Läuchli, E. J. Bergholtz, J. Suorsa, and M. Haque, Phys. Rev. Lett. 104, 156404 (2010). R. Thomale, D. P. Arovas, and B. A. Bernevig, Phys. Rev. Lett. 105 116805(2010). Z. Papic, B. A. Bernevig, and N. Regnault, Phys. Rev. Lett. 106, 056801 (2011). N. Regnault, B. A. Bernevig, F. D. M. Haldane, Phys. Rev. Lett. 103, 016801 (2009). X. Qi, H. Katsura, A. W. W. Ludwig, Phys. Rev. Lett. 108, 196402 (2012). F. Pollmann, A. M. Turner, E. Berg, and M. Oshikawa, Phys. Rev. B, 81, 064439 (2010). N. Bray-Ali, L. Ding, and S. Haas, Phys. Rev. B 80, 180504(R) (2009). A. M. Turner, Y. Zhang, A. Vishwanath, Phys. Rev. B, 82, 241102R (2010). A. M. Turner, Y. Zhang, R. S. K. Mong, A. Vishwanath, Phys. Rev. B 85, 165120 (2012). M. Kargarian, G. Fiete, Phys. Rev. B, 82, 085106 (2010). E. Prodan, T. L. Hughes,B. A. Bernevig, Phys. Rev. Lett. 105, 115501 (2010). T. L. Hughes, Phys. Rev. B 83, 245132 (2011). L. Fidkowski, Phys. Rev. Lett. 104, 130502 (2010). L. Fidkowski, T. S. Jackson and I. Klich, Phys. Rev. Lett. 107, 036601 (2011). J. Dubail and N. Read, Phys. Rev. Lett. 107, 157001 (2011). R. Thomale, A. Sterdyniak, N. Regnault, and B. A. Bernevig, Phys. Rev. Lett. 104, 180502 (2010).

[4] V. Alba, M. Haque, and A. M. Läuchli, Phys. Rev. Lett. 108, 227201 (2012).

[5] B. Nienhuis, M. Campostrini, and P. Calabrese, J. Stat. Mech. P02063 (2009).

[6] D. Poilblanc, Phys. Rev. Lett. 105, 077202 (2010).

[7] J. Schliemann, Phys. Rev. B 83, 115322 (2011).

[8] X. Deng and L. Santos, Phys. Rev. B 84, 085138 (2011).

[9] J. I. Cirac, D. Poilblanc, N. Schuch, and F. Verstraete, Phys. Rev. B 83, 245134 (2011).

[10] P. Calabrese and A. Lefevre, Phys. Rev A 78, 032329 (2008).

[11] E. Ercolessi, S. Evangelisti, F. Franchini, and F. Ravanini, Phys. Rev. B 83, 012402 (2011).

[12] E. Ercolessi, S. Evangelisti, F. Franchini, and F. Ravanini, Phys. Rev. B 85, 115428 (2012). 
[13] L. Banchi, F. Colomo, P. Verruchi, Phys. Rev. A 80, 022341 (2009).

[14] H. J. Lipkin, N. Meshkov, and A. J. Glick, Nucl. Phys. 62188 (1965).

[15] N. Meshkov, A. J. Glick, and H. J. Lipkin, Nucl. Phys. 62199 (1965).

[16] A. J. Glick, H. J. Lipkin, and N. Meshkov, Nucl. Phys. 62211 (1965).

[17] J. Latorre, R. Orus, E. Rico, and J. Vidal, Phys. Rev. A 71, 064101 (2005).

[18] R. Richardson, Phys. Lett. 3, 277 (1963).

[19] V. Popkov and M. Salerno, Phys. Rev A 71, 012301 (2005).

[20] V. Popkov and M. Salerno, Phys. Rev E 82, 011142 (2010).

[21] O. A. Castro-Alvaredo and B. Doyon, J. Stat. Mech. 1102:P02001 (2011).

[22] O. A. Castro-Alvaredo and B. Doyon, Phys. Rev. Lett. 108, 120401 (2012).

[23] G. Szego, Orthogonal polynomials (Amer. Mathematical Society, 1939), ISBN 0821810235.

[24] F. C. Alcaraz, M. N. Barber, M. T. Batchelor, R. J. Baxter, and G. R. W. Quispel, J. Phys. A: Math. Gen. 206397 (1987). E. K. Sklyanin, J. Phys. A: Math. Gen. 212375 (1988). 